\title{
(2) OPEN ACCESS \\ Patients' and health professionals' perceptions of the LoDED (limit of detection and ECG discharge) strategy for low-risk chest pain management: a qualitative study
}

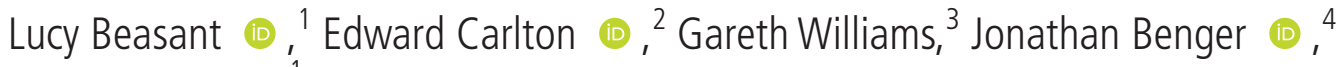 \\ Jenny Ingram
}

Handling editor Margaret Samuels-Kalow

${ }^{1}$ Bristol Medical School, University of Bristol, Bristol, UK 2Emergency Department, North Bristol NHS Trust, Westbury on Trym, UK

${ }^{3}$ Patient and Public Lead for the LoDED Trial, Swansea, UK

${ }^{4}$ Faculty of Health and Applied Sciences, University of the West of England, Bristol, UK

\section{Correspondence to}

Dr Edward Carlton, Emergency Department, North Bristol NHS Trust, Westbury on Trym, UK; eddcarlton@gmail.com

Received 15 February 2020 Revised 14 October 2020

Accepted 28 October 2020

Published Online First

9 December 2020

\section{Check for updates}

(c) Author(s) (or their employer(s)) 2021. Re-use permitted under CC BY-NC. No commercial re-use. See rights and permissions. Published by BMJ.

To cite: Beasant $L$

Carlton E, Williams G,

et al. Emerg Med J

2021:38:184-190

\section{ABSTRACT}

Background Rapid discharge strategies for patients with low-risk chest pain using high-sensitivity troponin assays have been extensively evaluated. The adherence to, and acceptability of such strategies, has largely been explored using quantitative data. The aims of this integrated qualitative study were to explore the acceptability of the limit of detection and ECG discharge strategy (LoDED) to patients and health professionals, and to refine a discharge information leaflet for patients with low-risk chest pain.

Methods Patients with low-risk chest pain who consented to a semi-structured interview were purposively sampled for maximum variation from four of the participating National Health Service sites between October 2018 and May 2019. Two focus groups with ED health professionals at two of the participating sites were completed in April and June 2019.

Results A discharge strategy based on a single undetectable hs-cTn test (LoDED) was acceptable to patients. They trusted the health professionals who were treating them and felt reassured by other tests, (ECG) alongside blood test(s), even when the clinical assessment did not provide a firm diagnosis. In contrast, health professionals had reservations about the LoDED strategy, including concern about identifying low-risk patients and a shortened patient observation period. Findings from 11 patient interviews and 2 staff focus groups (with 20 clinicians) centred around three overarching themes: acceptability of the LoDED strategy, perceptions of symptom severity and uncertainty, and patient discharge information.

Conclusion Rapid discharge for low-risk chest pain is acceptable to patients, but clinicians reported some reticence in implementing the LoDED strategy. Further work is required to optimise discharge discussions and information provision for patients.

\section{INTRODUCTION}

High-sensitivity troponin (hs-cTn) testing has led to an increasing drive to discharge low-risk patients who have presented to the emergency department (ED) with suspected cardiac chest pain soon after chest pain onset. ${ }^{1}$ We recently reported a pragmatic multicentre randomised controlled trial (RCT) which evaluated the clinical effectiveness of a single hs-cTn taken on arrival in the ED, in combination

\section{Key messages}

What is already known on this subject

- High-sensitivity cardiac troponin assays can facilitate the very early discharge of patients with chest pain from the ED.

- Adherence to rapid rule-out strategies has been previously explored in interventional trials using quantitative data.

What this study adds

- A discharge strategy based on a single undetectable high-sensitivity troponin test is acceptable to patients with low-risk chest pain.

- Patients trust the clinicians' decisions and accept the lack of a firm diagnosis; they also appreciate written discharge information.

- Health professionals are uncertain about the accurate identification of low-risk patients and concerned that the shortened observation period, associated with a single undetectable high-sensitivity troponin rule-out strategy, could lead to the early discharge of high-risk patients.

with a non-ischaemic ECG and irrespective of chest pain onset time (the limit of detection and ECG discharge strategy: LoDED). ${ }^{2}$ This strategy facilitated safe early discharge in over $40 \%$ of patients with low-risk chest pain.

Previous interventional studies evaluating novel rapid discharge strategies have demonstrated poor adherence by clinicians when embedded within usual care, reducing their clinical effectiveness. ${ }^{34}$ Adherence to a diagnostic strategy is clinician led and largely driven by concerns around risk and safety. ${ }^{4}$ Such barriers to effective implementation require further exploration. ${ }^{4}$ Quantitative data from trials demonstrate poor adherence and fail to explain why this occurs in practice. ${ }^{4-7}$

Acceptability of rapid discharge strategies is a shared construct between clinicians and patients and may influence adherence. ${ }^{5}$ It is known that acceptability can be improved using a shared decision-making model where written information provided to patients can improve patient knowledge, reduce decisional conflict and increase trust 
with a consequent decrease in downstream resource use. ${ }^{4-7}$ Exploring acceptability of a rapid discharge strategy with both clinicians and patients is likely to illuminate adherence and clinical practice. Written discharge information has been shown to reduce anxiety in patients with acute chest pain and may consequently improve acceptability. ${ }^{8}$ However up-to-date research is required to refine discharge information in the era of hs-cTn testing. ${ }^{1}$

This integrated qualitative study aimed to explore the acceptability of the LoDED strategy to patients and health professionals involved in the trial and to refine discharge information for patients with low-risk chest pain.

\section{METHODS}

\section{Patient semi-structured interviews}

Detailed inclusion and exclusion criteria for the LoDED trial have been published previously. ${ }^{9}$ Patients were eligible to participate in semi-structured telephone interviews if they attended the ED with chest pain, and their initial hs-cTn result was below the limit of detection (LoD), that is, those patients whose care would potentially be altered by trial enrolment and who were assessed as being low risk of subsequent ( 30 days) major adverse cardiac events. Low-risk patients in both the LoDED intervention and usual care arms were eligible for interview if they had consented to enrolment in the main RCT. Consent to contact for the interview was recorded on a central database, and the qualitative researcher contacted patients from six of the eight National Health Service (NHS) trial sites. Participants were contacted to take part in an interview within 90 days of index admission but after the 30 days outcome follow-up for the main RCT had been completed. Participants were purposively sampled for maximum variation (age, gender, and socioeconomic status). Most patients were selected from the LoDED intervention arm to explore acceptability of the new early discharge strategy. Two patients from the usual care arm were also interviewed to explore views about usual practice. Interviews explored experiences of patients' stay in the ED, acceptability of early discharge, positive and negative aspects of their ED care, and their views on the discharge information leaflet for patients with low-risk chest pain. Interviews were conducted with participants from four NHS trial sites.

\section{Healthcare professional focus groups}

Focus groups were conducted with health professionals from two of the eight participating trial sites. Both sites used serial hs-cTn testing over 2-3 hours as a control diagnostic strategy during the RCT. Health professionals were purposively selected for maximum variation in terms of gender, clinical background and varied levels of seniority. We also included general practitioners (GPs) working in the ED to gain a primary care perspective. All participating health professionals provided written consent and had recruited and/or treated patients during the trial. The focus groups aimed to explore their views about identifying, treating and discharging patients identified as low risk through the LoDED strategy, and their views on the discharge information leaflet for patients with low-risk chest pain.

Separate topic guides were used for the interviews and focus groups; these were developed in collaboration with Patient Advisory Group (PAG) members prior to data collection. LB conducted all the interviews and JI facilitated the focus group discussions with support from LB and EC.

\section{Patient discharge information leaflet}

Prior to trial commencement, we designed a bespoke patient discharge information leaflet with input from our PAG. This included a risk heuristic adapted from a previous interventional trial exploring shared decision-making, ${ }^{10} 11$ together with information on symptoms of myocardial infarction, the testing that was undertaken and 'safety-netting' advice. Refinement of discharge information drew on data from the interviews and focus groups. The discharge leaflet was given to all patients and health professionals prior to interview and focus group discussions. Detailed feedback was solicited during interviews and focus groups, particularly in relation to patients' understanding of the risk heuristic, and whether changes should be made to improve the leaflet. PAG members also reviewed and commented on amendments made to the leaflet after the qualitative feedback provided by patients (via semi-structured interviews) and health professionals (via focus groups). Figure 1 shows the final amended leaflet.

\section{Data analysis}

Interviews and focus groups were recorded and transcribed verbatim by a professional transcription service. Thematic analysis of the data was an ongoing and iterative process using NVivo software to organise and code the transcripts. ${ }^{12}$ Transcripts were initially coded by the qualitative researcher (LB). Codes and themes were developed and discussed with the lead qualitative researcher (JI) at regular intervals during data collection and analysis to achieve consensus. Two interview transcripts were also read and coded by an independent qualitative researcher (DJ) to compare and discuss the coding framework. Framework analysis was used to compare codes and themes across the patient and health professional data sets, encompassing similar (and contrasting) themes and findings. ${ }^{13}$ These approaches were inductive since they did not use predefined coding categories.

\section{Patient and public involvement}

The RCT had a PAG comprising four members who met on four occasions to advise the management team. The PAG were consulted at the point of analysis and interpretation of the data from this study, with findings presented in lay terms at a PAG meeting. They also gave detailed feedback on the revised version of the patient discharge leaflet. The patient and public involvement lead is a co-author of this paper.

\section{RESULTS}

\section{Patient semi-structured interviews}

One hundred and seven low-risk patients consented to contact and 52 were contacted by the qualitative researcher. Of those contacted, 11 patients from 4 sites were interviewed between October 2018 and May 2019: 9 patients from the LoDED intervention arm and 2 from the usual care arm. Six interviewees were women (54.5\%); mean age was 53 years. Patients who took part in interviews were slightly older than the low-risk patient subgroup who participated in the main RCT. Five participants were from the least deprived areas (deprivation measured by postcode), four from areas of high deprivation and two from areas with average deprivation in the UK (indices of multiple deprivation decile). ${ }^{1415}$ Interviews were conducted at a median of 72 days (IQR 65-78 days) post initial ED attendance. Table 1 shows demographic details for those interviewed compared with all low-risk patients in the LoDED cohort. 


\section{YOUR CHEST PAIN}

Chest pain is very common and can be caused by a number of reasons, many of which are not serious. For more information on chest pain visit: www.nhs.uk/conditions/chest-pain or use the QR code

\section{WHAT TESTS HAVE WE DONE?}

- A blood test, called troponin, to check whether there are any signs of heart muscle damage in your blood.

In your case, we have not detected any signs of heart muscle damage. This means it is very unlikely that you have had a heart attack

- A heart trace (ECG) to check the activity of the heart and to check that the heart is getting enough oxygen.

In your case your heart trace is normal.

\section{WHAT HAPPENS NOW?}

We think it is safe for you to go home.

If you have chest pain again that worries you or if any of your symptoms return or change, then call 999 or come back to the emergency department.

Following your visit, it is important to stay healthy to help your recovery. This includes stopping smoking if you smoke, reducing the amount of alcohol you drink, exercising and eating healthily. Your GP can help you with this.

\section{YOUR PERSONAL RISK}

The chance that you have had a heart attack is very low at less than $1 \%$ :

For every 100 people like you, who come into the emergency department with chest pain, it has been found that, after normal troponin blood tests and heart tracings, 99 people will not have had a heart attack. Therefore, we do not think that you have had a heart attack today, although we cannot rule out the possibility of heart complications in the future.
FURTHER INFORMATION:

NHS Choices website on chest pain: www.nhs.uk/conditions/chest-pain
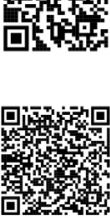

British Heart Foundation website for information about heart attacks and symptoms: www.bhf.org.uk/ informationsupport/conditions/heartattack/heart-attack-symptoms

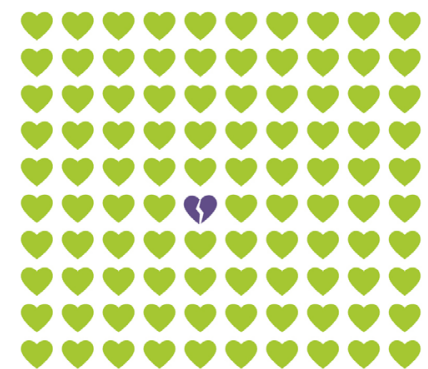

Figure 1 Leaflet for low-risk chest patients to take home following discharge. NHS, National Health Service.

\section{Healthcare professional focus groups}

Twenty ED health professionals consented to involvement in two focus groups, which were conducted in April and June 2019. Focus groups were scheduled on days when a varied group of health professionals were available, incorporating a wide range

Table 1 Demographics and clinical characteristics of patients with an initial high-sensitivity troponin (hs-cTn) level below the limit of detection (LOD) in the randomised controlled trial cohort and qualitative interview sample

\begin{tabular}{lll}
\hline & $\begin{array}{l}\text { All low-risk* trial } \\
\text { patients } \mathrm{n=274}(\%)\end{array}$ & $\begin{array}{l}\text { Interview patients } \\
\mathrm{n}=11(\%)\end{array}$ \\
\hline LoDED intervention arm & $141(51.5 \%)$ & $9(81.8 \%)$ \\
Mean age & 46 years & 53 years \\
Gender: female & $144(52.5 \%)$ & $6(54.5 \%)$ \\
Gender: male & $130(47.5 \%)$ & $5(45.5 \%)$ \\
Ethnic origin: white & $228(84 \%)$ & $11(100 \%)$ \\
Chest pain history (clinician reported) & \\
Slightly suspicious & $175(64 \%)$ & $6(54.6 \%)$ \\
Moderately suspicious & $77(28 \%)$ & $4(36.4 \%)$ \\
Highly suspicious & $22(8 \%)$ & $1(9 \%)$ \\
Successful early discharget & $165(61.3 \%)$ & $9(82 \%)$ \\
Median LOS hours (IQR) $\dagger$ & $3.7(2.8-5.2)$ & $3.5(2.7-3.8)$ \\
\hline
\end{tabular}

* Low-risk patients were those with an initial hs-cTn below the LoD and a non ischaemic ECG in either trial arm.

†Successful early discharge data available for 269/274 low-risk patients. łLength of stay (LOS; hours) data available for 268/274 low-risk patients. LoDED, limit of detection and ECG discharge strategy. of experience in emergency care, nursing, and GPs working in the ED. Some staff travelled to attend on a non-working day and cover was provided for others to attend, thus ensuring that the groups were representative. Table 2 provides details of those who participated in focus groups, including their gender and job title.

Thematic analysis: patient and health professional themes and sub-themes

Three overarching themes were identified, describing both patient and health professionals' acceptability of the LoDED strategy (table 3 ). In the quotations, patients are identified by ID number, gender, age, and study arm (LoDED intervention or usual care) and health professionals by ID number, broad job title, gender and focus group site.

Table 2 Demographics of health professionals who took part in focus groups

\begin{tabular}{lll}
\hline & $\begin{array}{l}\text { Focus group } 1 \mathrm{n}=9 \\
(\%)\end{array}$ & $\begin{array}{l}\text { Focus group } \\
\mathbf{2 n = 1 1 ( \% )}\end{array}$ \\
\hline Gender: female & $3(33.3 \%)$ & $7(63.7 \%)$ \\
Gender: male & $6(66.7 \%)$ & $4(36.3 \%)$ \\
ED doctors (varied levels of seniority) & $5(55.6 \%)$ & $8(72.7 \%)$ \\
\hline Nursing staff & $3(33.3 \%)$ & $1(9.1 \%)$ \\
GPs & $1(11.1 \%)$ & $1(9.1 \%)$ \\
Healthcare assistant & 0 & $1(9.1 \%)$ \\
\hline
\end{tabular}

Beasant L, et al. Emerg Med J 2021;38:184-190. doi:10.1136/emermed-2020-209539 
Table 3 Patient and health professional themes and sub-themes

\begin{tabular}{lll}
\hline Theme & Patients & Health professionals \\
\hline $\begin{array}{l}\text { Acceptability of single blood test } \\
\text { (LoDED) strategy }\end{array}$ & $\begin{array}{l}\text { Trust and reassurance } \\
\text { Having trust in the treating health professionals } \\
\text { Feeling reassured by the number of other tests alongside blood } \\
\text { test(s) } \\
\text { Practicalities and reduced anxiety }\end{array}$ & $\begin{array}{l}\text { Reservations and anxieties } \\
\text { Identifying low-risk patients } \\
\text { Lack of clinician experience } \\
\text { Shortened observation period }\end{array}$ \\
$\begin{array}{l}\text { Perceptions of symptom severity and } \\
\text { uncertainty }\end{array}$ & $\begin{array}{l}\text { Identification and experience of chest pain } \\
\text { Lack of a firm diagnosis } \\
\text { Understanding of being a low-risk chest pain patient } \\
\text { Legitimate use of NHS services }\end{array}$ & $\begin{array}{l}\text { Ambiguity: diagnosis and ongoing management of 'low-risk' } \\
\text { chest pain symptoms } \\
\text { Explaining risk and uncertainty to low-risk patients } \\
\text { Acceptance: a small number of low-risk patients may have a heart } \\
\text { attack in the future } \\
\text { Lack of follow-up after ED attendance }\end{array}$ \\
& $\begin{array}{l}\text { Lack of current discharge information for patients with low- } \\
\text { risk chest pain }\end{array}$ \\
Patient discharge information & $\begin{array}{l}\text { Patients valued written information } \\
\text { Readability and clarity }\end{array}$ & Fills anformation gap for low-risk chest pain patients \\
\hline
\end{tabular}

LODED, limit of detection and ECG discharge strategy.

\section{Theme 1: acceptability of single blood test (LoDED) strategy Trust and reassurance}

Most patients stated that they felt the LoDED strategy was acceptable and this was influenced by having trust in the treating health professionals, feeling reassured by other tests, such as an ECG, and the practicalities associated with having blood tests taken:

If this is the way things work [one blood test] they know in the hospital better than we do. I thought it was quite a good idea to do that... I felt comfortable that people knew what they were doing (Patient_27, Female, 69 years, LoDED Intervention arm)

After the ECG I was absolutely fine, because they told me that's fine, it must be something else (Patient 25, Male, 45 years, LoDED Intervention arm)

Some patients highlighted that more time spent 'hanging around' in the ED was potentially stressful for them and for their wider family, and a 'waste of time' if they had been identified as being in a low-risk group:

To wait potentially for another two hours when the first results come back as under the risk level seems that's more time for you to sit and worry... if you have to keep doing another set of tests just to quantify something when your first test already comes out as low it seems a bit of a waste of everybody's time, and resources really (Patient_23, Female, 58 years: LoDED Intervention arm)

Health professionals acknowledged that having serial blood tests could be anxiety provoking for patients who had chest pain, and adapted to the LoDED strategy by rationalising that most patients would be unaware of a change from serial sampling to a single test strategy (LoDED):

...you're confident that first blood test is okay, then should we be really offering this kind of... [two blood tests] I think especially the group of patients that may come in with chest pains is anxiety related, we are just going to add to that... (ED nurse_7, Female: Focus group 1)

I don't necessarily think that patients have got the expectation of serial investigation. So they come in and have some tests done which is what they are expecting, but then I don't think they are planning on spending several hours hanging around in the emergency department ... (ED doctor_4, Male: Focus group 1)

\section{Reservations and anxieties}

In contrast to patients, it was health professionals who had reservations about moving towards a single test strategy, despite attempts to rationalise and adapt to the single test strategy. Health professionals pointed out that they felt uncomfortable with the LoDED strategy because it was a change in practice. However, two health professionals saw the LoDED strategy as a progression in the field of medicine, highlighting the way in which testing evolves and changes over time in parallel with career progression:

The evolution of testing throughout my career and how things have changed and when one test replaced another test, it's not like the patients are coming in having no investigations done, they are going to have as a minimum if we're wanting to rule out cardiac related chest pain an ECG, and blood tests (ED doctor_4, Male: Focus group 1)

I think it probably makes clinicians feel more uncomfortable than the actual patients, ... because most patients aren't going to know, and it's not like we're still not being just as thorough, it's just a different way of monitoring (GP_3, Female: Focus group 1)

Most health professionals highlighted some reservations or unanswered questions about using the one blood test pathway and reliance on tests in a 'usual care' environment. Reservations largely centred around how to identify and differentiate between low-risk, medium-risk and high-risk patients:

For me it's about properly identifying a low-risk patient group in whom we know there's a less than $5 \%$ risk of them having an acute coronary syndrome before you ever do a troponin. So if you've already identified the right group then I would be happy with the LoDED protocol.... One of my concerns is that there will be admission creep and actually we've started to put people on it who are nowhere near low risk, their history of ischaemic heart disease, their ECG looks fine, they presented with discrete chest pain and they are not in pain anymore (ED doctor_18, Male: Focus group 2)

A reservation in relation to the LoDED strategy was its use by those with limited clinical experience (in years and expertise) who might rely too heavily on one blood test, further impacted by the shortened observation period associated with the pathway that could lead to early discharge of a high-risk patient:

I've just got a niggling concern that sometimes chest pain is viewed as, so long as your troponin is negative then you're home and dry, $\ldots$ and currently I guess with the sixhour pathway we have this window to observe the patient on the CDU... to give the junior clinician some time to see that patient and let those diagnostic thoughts happen (ED doctor_14, Male: Focus group 2)

In contrast, it was also pointed out that a lack of continuity of care associated with multiple diagnosing and treating health professionals using the serial testing strategy, could contribute to missing a serious diagnosis and early discharge:

I am not sure to what degree they get a thoughtful review with the second one [troponin test result]. I suspect if we make the dis- 
charge decision when the patients actually are in front of us in the department... that might be an advantage of doing it sooner. At the moment they do get quite dislocated from the clinician who made the initial assessment... (ED doctor_19, Male: Focus group 2)

\section{Theme 2: perceptions of symptom severity and uncertainty Identification and experience of chest pain}

Patients described uncertainty about the severity of their symptoms, and whether or not pain was indicative of myocardial infarction or something less serious. All had experienced some form of chest, muscle pain or 'tightness' in the chest; some also reported a cold sweat, dizziness and feeling sick; patients carried out their own 'risk assessment' with some deciding to go to the ED themselves, others were told by family or colleagues that they should attend:

I came into work and I had chest pains, and it was peculiar because it didn't really feel like anything I had felt before, and I spoke to some people at work about it and... went through this first aiders test and they said the best thing to do really is just to go into ED... (Patient_12, Male, 50 years: LoDED Intervention arm)

I just came over a little bit dizzy, felt a little bit sick, so I just stopped and leaned over the trolley and it passed away, and I thought at the time actually it was more a panic thing rather than anything else, but obviously the pain was worrying me so then I decided I had better do something about it... (Patient_27, Female, 69 years: LoDED Intervention arm)

Uncertainty was also reflected in patients' diagnosis when they attended the ED. Most patients were not given a firm diagnosis after their ED assessment. Some were provided with 'potential' non-cardiac diagnoses (eg, anxiety, muscle pain); others were not, but they accepted this either because their symptoms had reduced during their time in the ED, or they drew their own conclusions about symptoms based on pre-existing health conditions (panic attack) or the ageing process:

He [the clinician] just seems to think it was muscle strain in the chest (Patient_29, Male, 37 years: Usual Care arm)

At the end of the time [in ED] I was more realistic in terms of the fact of thinking it was a panic attack... if I hadn't realised that then I probably would have been a bit more anxious (Patient_23, Female, 58 years: LoDED Intervention arm)

I thought well I'm getting older now, maybe this is what happens as you get older... aches and pains about something that's nothing (Patient_12, Male, 50 years: LoDED Intervention arm)

None of the patients recalled explicit conversations about their current 'risk' or specifically being a low-risk patient, but having received the discharge leaflet some perceived that they were not 'at risk' and knew what to do if they had similar symptoms in the future:

Only in terms of the fact that the results came back that they didn't think I was a risk... just to give me the leaflet and say if I did have any... if I did start to have any symptoms etc, then to just [do] what I had done before and call (Patient_23, Female, 58 years: LoDED Intervention arm)

LoDED intervention patients consistently highlighted the importance of appropriate and legitimate NHS use, they did not want to waste health professionals time, or use NHS resources unnecessarily. The rapid rule out strategy reinforced cost effective use of time and NHS resource:

I didn't want to waste their time at the end of the day, I just wanted a quick answer, but they were very thorough in they weren't going to just let me leave after an hour, they said no we need to check this (Patient_6, Male 53 years: LoDED Intervention arm)

you didn't have to do a second blood test, it's a waste of time and money (Patient_25, Male 45 years: LoDED Intervention arm)

\section{Ambiguity: diagnosis and ongoing management of 'low-risk' chest pain symptoms}

Some health professionals discussed the reasons why they could not always give a firm diagnosis when providing explanations for chest pain, specifically when ECG and troponin tests are normal. They felt they often discussed this diagnostic ambiguity with low-risk patients:

when it comes to discharge explanations it's often that we don't give a diagnosis, and I tend to use the ' we have ruled out this, this and this, and it is probably just something muscular and you don't need to worry about it. But of course it's a safety netting spiel here..." (ED doctor_4, Male: Focus group 1)

I think people don't like being sent away without absolute it's this, and we don't always know, and sometimes we have to say we don't know but we've ruled out these emergency situations... (ED doctor_5, Female: Focus group 1)

Nursing staff from one focus group reported that patients often wanted more explanation when they have been told by a doctor that their condition was 'not cardiac':

I think from the nursing point of view quite often once the doctor has been in there and told them that it's not cardiac,... they will be saying to us, "what is it then?" and I just usually say well, we're pretty sure that it's not cardiac related. But quite often, especially with the older people, don't like to ask the doctors questions so they will ask us (ED nurse_7, Female: Focus group 1)

A research nurse from the second focus group felt that she simply reiterated 'what was happening' in 'layman's terms':

So I think all I probably did was reiterating in slower terms maybe more layman's terms what the process is that they are going through... and confirmed for them what's happening (Research nurse_13, Female: Focus group 2)

Some health professionals reported a lack of follow-up for patients with low-risk chest pain after discharge from the ED. Although EDs routinely transfer records to a patient's GP informing them that they have attended for chest pain, many acknowledged that these patients would not be formally followed up in primary care and the onus would be on the patient to make an appointment with their GP after attending the ED should they believe it necessary to do so:

Sometimes we do get patients who have been to ED with chest pain and then no follow-up, ...... and they get sent back to GPs as the mop it up... (GP_3, Female: Focus group 1)

The wider impact of a one test strategy was discussed in the context of; lack of resource in primary care to follow up patients with low-risk chest pain, strain on NHS resources, changes in the way the general public use healthcare and a changing distribution of workload across the NHS:

I see a lot of chest pain in GP world... I think GPs are very stretched at the moment and a lot of people are leaving general practice because they are seeing a patient every ten minutes, I think it's very difficult to take a chest pain history in ten minutes and do everything, I don't have the resources (GP_3, Female: Focus group 1)

We do know that [ED] crowding kills, and it is really important, and the ambulance service is hugely stretched, and if we're sending the message that if you get any kind of chest pain, you ring 
an ambulance because this might be really serious, and you go to ED... even if you are sending them home early... what risk are you putting on everyone else in that department (ED doctor_4, Male: Focus group 1)

\section{Theme 3: patient discharge information \\ Patients valued written information}

Most patients appreciated having written information they could take with them when they left the ED, to read in their own time. The discharge leaflet was clear, easy to understand and reassured most patients. Many patients did not recall any discussion of the leaflet with the clinical team at discharge and could not find, or had not kept, a copy of the leaflet they had been given at discharge. One patient gave the leaflet to their partner to read, but most did not share the information with other members of their immediate family:

I think it was just the fact that it was really well written, and it put your mind at rest, because there's going to be a lot of people including myself that are thinking I don't know. But I really read it in- depth (Patient_15, Female, 56 years: LoDED Intervention arm)

I think it's better to have a leaflet than just verbal, because I think it cements it in a little bit better than just someone's... what feels like someone's opinion if you like (Patient_12, Male, 50 years: LoDED Intervention arm)

Patients felt that the leaflet conveyed an acceptable level of risk, provided contact information should they need it, and felt reassured that they were not likely to have a myocardial infarction in the near future:

The takeaway [message] for me was everything is fine, and then almost instantly my brain let it go... I look at it as $1 \%$ is like zero really, I am in the clear, that's the way I felt about it (Patient_12, Male, 50 years: LoDED Intervention arm)

\section{Lack of current discharge information for patients with low-risk chest pain}

Feedback about the discharge leaflet from health professionals was largely positive; they felt it was appropriate for future use in the general population of patients with low-risk chest pain. Health professionals liked the layout, the 'clear' risk heuristic and highlighted the lack of existing written information available in routine care for patients with low-risk chest pain:

I think it's interesting that we don't hand out discharge information. We do for so many other things, pretty much every condition that comes through minors has got it, but here is this potentially life-threatening problem on the way in, and we don't give them anything to say this is what the doctor has assessed so what we think (ED doctor_4, Male: Focus group 2)

Health professionals offered some useful suggestions regarding the wording and layout of the leaflet, including the addition of $\mathrm{QR}$ codes and links to national websites. A revised version of the leaflet was reviewed by our PAG to finalise the content and layout. Minor changes were made to wording to clarify the advice and the final version is shown in figure 1 .

\section{DISCUSSION}

This qualitative study has shown that a discharge strategy based on a single undetectable hs-cTn test (LoDED) was acceptable to patients. Patients trusted the clinicians' decision and were reassured by the other tests carried out. They also accepted the lack of a firm diagnosis, understood that they were at 'low-risk', did not want to waste NHS resources and appreciated the written leaflet given on discharge. Health professionals were uncertain about the accurate identification of low-risk patients and concerned that the shortened observation period associated with the LoDED strategy could lead to the early discharge of high-risk patients. However, some suggested that an informed discharge discussion is important, and facilitates safe early discharge. Positive framing and information provision around normal test results has been shown to improve reassurance, yet this issue remains underexplored in emergency medicine literature. ${ }^{16}$

Health professionals also highlighted pressure placed on the ED due to increased patient numbers and a lack of follow-up for patients with low-risk chest pain after ED discharge due to current resource pressures in primary care. However, the LoDED RCT found that up to $50 \%$ of low-risk patients in both trial arms visited their GPs within 30 days of attending the ED. ${ }^{2}$ This may have been due to the lack of a firm ED diagnosis for their chest pain which concerned patients and health professionals alike. Previous research has highlighted that ED health professionals tend to focus on the exclusion of cardiac-related causes of chest pain, rather than the 'positive management of non-cardiac chest pain'. ${ }^{17}$ While reassurance may be important, it may be ineffective for patients with non-cardiac chest pain. ${ }^{18}$ Further interventions such as brief cognitive therapy have been evaluated but are not recommended in unselected non-cardiac chest pain patients. ${ }^{18}$ Therefore, further mixed-methods research is required to establish the optimal approach to discharge discussions, shared decision-making and information provision in this patient group.

This analysis also raises other important considerations for the clinical implementation of very rapid rule-out strategies based on hs-cTn testing. These include an over reliance on the hs-cTn test itself, a lack of consideration of alternative serious diagnoses and the clinical experience of clinicians making the discharge decision. The diagnostic literature in this field tends to recruit only patients in whom an acute coronary syndrome is suspected and excludes patients in whom an alternative serious diagnosis is suspected; as a result these issues are poorly understood. One exploratory study has shown improved diagnostic work-up when patients are assessed by more senior ED clinicians, ${ }^{19}$ and to mitigate these issues we therefore recommend that a robust "senior sign-off" process is put in place, as proposed by the Royal College of Emergency Medicine. ${ }^{20}$

A limitation of this qualitative study is the small sample size; however, no new themes were arising from the data and saturation was reached. Although a high proportion of low-risk patients were contacted to take part in the qualitative study (48.6\% of those who provided consent), only $21.2 \%$ agreed to an interview. Interviews were conducted after the 30 days outcome follow-up had been completed, but on average low-risk patients completed this outcome measure 67.7 days after they attended the ED. Patients therefore took part in interviews more than 2 months after trial participation, and this may have negatively impacted patients' willingness to participate in an interview and recall the details of their attendance. A limiting factor that may have introduced bias to the focus group sample was that work commitments may have limited health professional's availability to attend on the scheduled day and time. Bringing professionals with different levels of power together in the same focus group (eg, consultants and nursing staff) may introduce power dynamics and bias (eg, nursing staff feel less empowered to air their views and opinions). However, members of nursing staff and the healthcare assistant who attended focus groups all aired their views and were not apprehensive about discussing their experience and views of LoDED. 


\section{CONCLUSIONS}

Rapid discharge in patients with low-risk chest pain is acceptable to patients, but clinicians reported some reticence in implementing the LoDED discharge strategy. Key factors in the successful implementation of rapid discharge strategies are likely to be (1) taking the time to discuss what it means to be 'lowrisk', (2) acknowledging and explaining diagnostic ambiguity to patients and (3) improved verbal and written information provision.

\section{Twitter Edward Carlton @eddcarlton}

Acknowledgements The authors are grateful to all the patients and staff involved in the interviews and focus groups and the Patient Advisory Group for their input. They also thank Debbie Johnson (DJ) for assisting with double coding the interviews and Hazel Taylor for providing quantitative data. The LoDED trial and this integrated qualitative study was funded by the National Institute for Health Research (NIHR) through its Research for Patient Benefit (RfPB) Programme (grant reference number PB-PG-0416-20012). Jonathan Benger is a NIHR Senior Investigator. The Peninsula Clinical Trials Unit at the University of Plymouth, provided support with the design, management and logistics of the trial. They are very grateful to their team, especially Sarah Campbell (Senior Trials Manager) and Rebecca Chapman (Trials Manager).

Contributors EC conceived the original idea for the study. JI supported by LB and EC developed the qualitative study design. LB undertook the interviews and JI led the focus groups with support from LB and EC. LB undertook the analysis, supported by JI. LB wrote the first draft of the paper. All authors contributed to the discussion and final paper. EC is the guarantor for the study.

Funding This research was funded by the National Institute for Health Research (NIHR) Research for Patient Benefit programme (PB-PG-0416-20012).

Disclaimer The views expressed are those of the author(s) and not necessarily those of the NIHR or the Department of Health and Social Care.

Competing interests None declared.

Patient and public involvement Patients and/or the public were involved in the design, or conduct, or reporting, or dissemination plans of this research. Refer to the Methods section for further details.

Patient consent for publication Not required.

Ethics approval The study was approved by the South West Frenchay Research Ethics Committee (REC reference 18/SW/0038) sponsored by North Bristol NHS Trust, with selected sponsorship activities formally delegated to the Peninsula Clinical Trials Unit (PenCTU). Trial registration: ISRCTN86184521: https://doi.org/10.1186/ ISRCTN86184521.

Provenance and peer review Not commissioned; externally peer reviewed.

Data availability statement All data relevant to the study are included in the article or uploaded as supplementary information.

Open access This is an open access article distributed in accordance with the Creative Commons Attribution Non Commercial (CC BY-NC 4.0) license, which permits others to distribute, remix, adapt, build upon this work non-commercially, and license their derivative works on different terms, provided the original work is properly cited, appropriate credit is given, any changes made indicated, and the use is non-commercial. See: http://creativecommons.org/licenses/by-nc/4.0/.

\section{ORCID iDs}

Lucy Beasant http://orcid.org/0000-0002-4279-5644

Edward Carlton http://orcid.org/0000-0002-2064-4618

Jonathan Benger http://orcid.org/0000-0001-6131-0916

Jenny Ingram http://orcid.org/0000-0003-2366-008X

\section{REFERENCES}

1 National Institute for Health and Care Excellence. Chest pain of recent onset: assessment and diagnosis (update). CG95. London: NICE, 2016.

2 Carlton EW, Ingram J, Taylor $\mathrm{H}$, et al. Limit of detection of troponin discharge strategy versus usual care: randomised controlled trial. Heart 2020;106:1586-94.

3 Al-Ani M, Winchester DE. Prevalence and overlap of noncardiac conditions in the evaluation of low-risk acute chest pain patients. Crit Pathw Cardiol 2015;14:97-102.

4 Musey PI, Kline JA. Emergency department cardiopulmonary evaluation of low-risk chest pain patients with self-reported stress and anxiety. J Emerg Med 2017; 52:273-9.

5 Musey PI, Patel R, Fry C, et al. Anxiety associated with increased risk for emergency department recidivism in patients with low-risk chest pain. Am J Cardiol 2018;122:1133-41.

6 Than MP, Pickering JW, Aldous SJ, et al. Effectiveness of EDACS versus adapt accelerated diagnostic pathways for chest pain: a pragmatic randomized controlled trial embedded within practice. Ann Emerg Med 2016;68:93-102.

7 Poldervaart JM, Reitsma JB, Backus BE, et al. Effect of using the heart score in patients with chest pain in the emergency department: a Stepped-Wedge, cluster randomized trial. Ann Intern Med 2017;166:689-97.

8 Arnold J, Goodacre S, Bath P, et al. Information sheets for patients with acute chest pain: randomised controlled trial. BMJ 2009;338:b541.

9 Carlton E, Campbell S, Ingram J, et al. Randomised controlled trial of the limit of detection of troponin and ECG discharge (LoDED) strategy versus usual care in adult patients with chest pain attending the emergency department: study protocol. BMJ Open 2018;8:e025339.

10 Hess EP, Hollander JE, Schaffer JT, et al. Shared decision making in patients with low risk chest pain: prospective randomized pragmatic trial. BMJ 2016;355:i6165.

11 Hess EP, Knoedler MA, Shah ND, et al. The chest pain choice decision aid: a randomized trial. Circ Cardiovasc Qual Outcomes 2012;5:251-9.

12 QSR International Pty Ltd. NVivo qualitative data analysis software; version 12, 2018

13 Gale NK, Heath G, Cameron E, et al. Using the framework method for the analysis of qualitative data in multi-disciplinary health research. BMC Med Res Methodol 2013;13:117.

14 Department for Communities and Local Government. The English indices of multiple deprivation (IMD) 2015. London, 2015.

15 Community safety and social inclusion: Stats Wales. The Welsh index of multiple deprivation (IMD), 2016

16 Petrie KJ, Müller JT, Schirmbeck F, et al. Effect of providing information about normal test results on patients' reassurance: randomised controlled trial. BMJ 2007;334:352.

17 Chambers JB, Marks EM, Hunter MS. The head says Yes but the heart says NO: what is non-cardiac chest pain and how is it managed? Heart 2015;101:1240-9.

18 Mulder R, Zarifeh J, Boden J, et al. An RCT of brief cognitive therapy versus treatment as usual in patients with non-cardiac chest pain. Int J Cardiol 2019;289:6-11.

19 Carlton EW, Than M, Cullen L, et al. 'Chest pain typicality' in suspected acute coronary syndromes and the impact of clinical experience. Am J Med 2015;128:1109-16.

20 Royal College of Emergency Medicine Quality in Emergency Care Committee. Consultant Sign-Off standard, 2016. Available: https://www.rcem.ac.uk/docs/ Consultants\%20Sign\%20off/Consultant\%20Sign\%200ff_June\%202016.pdf 